\title{
Self-optimizing Uplink Outer Loop Power Control for WCDMA Network
}

\author{
Angelo Gary MARKOC ${ }^{1}$, Gordan SISUL ${ }^{2}$ \\ ${ }^{1}$ Radio Access Network Development Dept., Vipnet d.o.o., Vrtni put 1, 10000 Zagreb, Croatia \\ ${ }^{2}$ Dept. of Wireless Communications, Faculty of Electrical Engineering and Computing, Unska 3, 10000 Zagreb, Croatia
}

a.gmarkoc@vipnet.hr, gordan.sisul@fer.hr

\begin{abstract}
The increasing demands for high data rates, drives the efforts for more efficient usage of the finite natural radio spectrum resources. Existing wideband code division multiple access (WCDMA) uplink outer loop power control has difficulty to answer to the new load on air interface. The main reason is that the maximum allowed noise rise per single user is fixed value. In worst case uplink load can be so high that all services, including conversational service, could be blocked. In this paper investigation has been performed to present correlation of main system parameters, used by uplink outer loop power control, to uplink load. Simulation has been created and executed to present difference in current implementation of uplink outer loop power control against proposed changes. Proposed solution is self-optimizing uplink outer loop power control in a way that maximum allowed noise rise per single user would be dynamically changed based on current uplink load on cell.
\end{abstract}

\section{Keywords}

WCDMA, outer loop power control, uplink received signal strength indicator (UL RSSI), maximum allowed signal to interference ratio (sirMax), transmission target error (TTE)

\section{Introduction}

Power control is essential in WCDMA systems to provide satisfactory quality of service (QoS) [1] and to combat several problems:

- To mitigate the fading effect.

- To adjust the radiated power of mobile stations in a way that all received signals at the base station have the same signal to interference ratio (SIR) for the same bit rate. This is necessary to prevent the socalled near far problem [2].

- To reduce co-channel interference by concurrent users. This has a direct impact on the capacity of cells in the uplink.
SIR in the WCDMA system corresponds to the ratio of the received useful signal at the receiver of base station over a frequency range of $5 \mathrm{MHz}$ width compared to interfering co-channel sources. In the uplink, received useful signal at the receiver of base station is signal from the mobile station. Sources of interfering signals are all other mobile stations in the cell, as well as other mobile stations in neighboring WCDMA cells that transmit on the same channel [3].

There are three types of algorithm for power control, which are implemented in WCDMA system: open loop power control, inner loop power control, outer loop power control. All three loops for power control work together during radio connection. Open loop power control is used to calculate the required radiated power by the mobile station for access preamble, the random access channel (RACH) and the initial power for dedicated channels (DCH) [4]. Once dedicated channels have been established outer and inner loop power control shall cooperate in order to maintain the necessary block error rate (BLER) for voice or TTE for data calls. This is done in a way that outer loop power control sets and adjusts the SIR target value used by inner loop power control [5]. Outer loop power control for uplink monitors the cyclic redundancy check (CRC) of transport blocks after diversity combining at the control station and change the SIR target value according to the SIR target control algorithms for the uplink. Thus maintains the desired TTE in the uplink, regardless of user's radio conditions, and whether user is stationary or mobile [6]. This way of cooperation between three types of power control algorithm has been presented at Fig. 1.

From Fig. 1, if TTE for the transmitting mobile station is not $\mathrm{OK}$, outer loop power control will increase SIR target [7], thus causing request in increase of uplink radiated power by inner loop power control. During a call, the SIR target varies between implemented lower (sirMin) and upper boundaries (sirMax) [8]. Maximum allowed increase in SIR target, thus maximum allowed increase of uplink radiated power per mobile station, is set by parameter sirMax on radio network controller (RNC). That means that every cell on every radio base station (RBS) controlled from same RNC have same sirMax value, regardless of different uplink traffic load. Setting of maximum allowed 

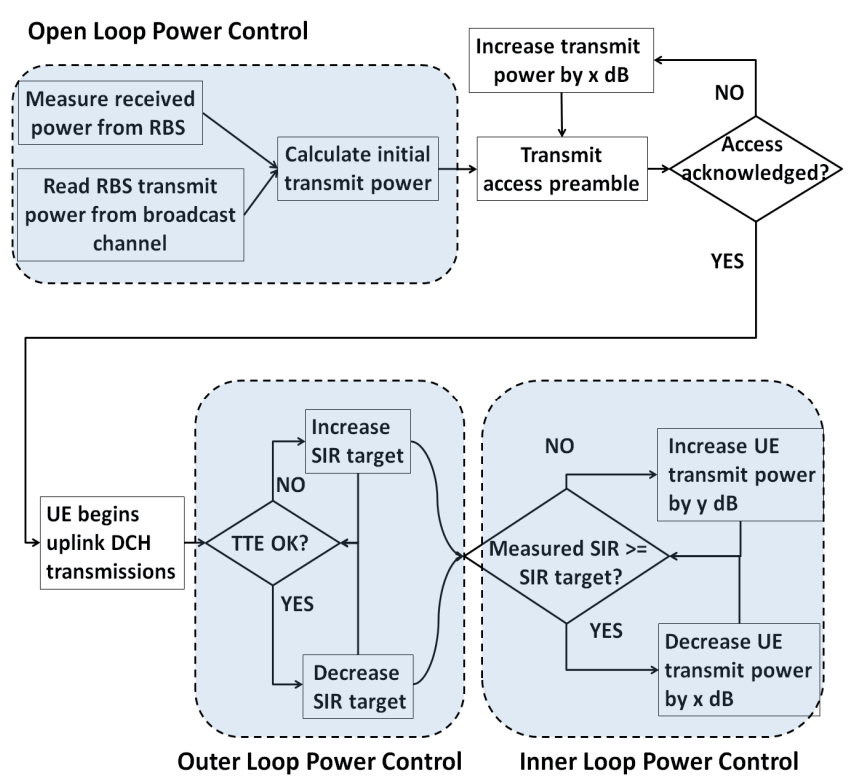

Fig. 1. Cooperation between three types of uplink power control algorithm

SIR target by sirMax parameter regardless of traffic load per cell can cause two types of problems:

- In scenario with a high number of users in the cell, sirMax value would be too high, thus causing too much radiated power from users and severe interference.

- In scenario with a low number of users in the cell, sirMax value would be too low, thus users served by that cell would not be able to exploit entire uplink cell capacity.

In this paper, problem with a high number of users in the cell has been analyzed in detail, but the proposed solution is applicable for both types of problem.

\subsection{Related Work}

One of the first solutions for uplink interference cancellation was enhanced receivers techniques presented at [9]. Increasing number of receiver antennas was recommended and investigated, like 4RX diversity [10], distributed antenna system (DAS) presented at [11] or directional antennas for wireless indoor solution presented at [12]. Furthermore, measurements in live WCDMA network [13] have been performed to propose solutions for WCDMA uplink air interface capacity increase. Many improvements have been developed to improve antennas, RBS processor units, or RBS power amplifiers and receivers. However, none of the implemented solutions have solved the problem with uplink interference. Even more, all new functionalities and improvements of hardware and software from RBS side implemented together haven't solved the problem.

\section{RNC Parameter Setting Impact on Uplink Capacity}

In this section investigation from lab environment has been presented to explain correlation between uplink noise rise and cell capacity according to sirMax and TTE setting on RNC. Uplink capacity is defined as maximum allowed noise rise over thermal noise due to interference at which it is still possible to distinguish users [14].

$$
U L R S S I_{\max }=U L R S S I_{0}+N R_{\max }
$$

where $U L R S S I_{\max }$ is uplink capacity, $U L R S S I_{0}$ is load of empty cell and $N R_{\max }$ is maximum allowed noise rise. If there are no users in the cell, and if the cell has not been interfered by transmitting mobile stations from neighboring base stations, the measured signal strength at the base station receiver (UL RSSI0) corresponds to thermal noise [15]:

$$
\begin{aligned}
& N=k \times T \times B[\mathrm{~W}]= \\
& =10 \log (k)+10 \log (T)+10 \log (B)[\mathrm{dBW}]
\end{aligned}
$$

where $k$ is Boltzmann constant, $T$ is temperature in Kelvin and $B$ is bandwidth in $\mathrm{Hz}$. For a temperature of $20^{\circ} \mathrm{C}$ and the bandwidth of $5 \mathrm{MHz}$, the obtained result is:

$$
U L \operatorname{RSSI}_{0}=N=-136.9 \mathrm{dBW}=-106.9 \mathrm{dBm} .
$$

Expressions (4)-(11) from [16] have been used to calculate noise rise, which is equal to:

$$
N R=-10 \times \log \left(1-\eta_{\mathrm{UL}}\right)[\mathrm{dB}]
$$

where $\eta_{\mathrm{UL}}$ is the uplink load factor. The uplink load factor can be calculated as a sum of load factors of all $M$ uplink connections $L_{\mathrm{j}}$ in a cell:

$$
\eta_{\mathrm{UL}}=\sum_{j=1}^{M} L_{\mathrm{j}} .
$$

The load factor of each connection is derived below. First the $E_{\mathrm{b}} / N_{\mathrm{o}}$, energy per user bit divided by the noise spectral density, is defined:

$$
\left(E_{\mathrm{b}} / N_{\mathrm{o}}\right)_{\mathrm{j}}=P G_{\mathrm{j}} \times \frac{S S_{\mathrm{j}}}{S S_{\text {other }}}
$$

where $P G_{\mathrm{j}}$ is processing gain of user, $S S_{\mathrm{j}}$ is signal strength of user and $S S_{\text {other }}$ is signal strength of other users. This can be written as:

$$
\left(E_{\mathrm{b}} / N_{\mathrm{o}}\right)_{\mathrm{j}}=\frac{W}{v_{\mathrm{j}} R_{\mathrm{j}}} \times \frac{P_{\mathrm{j}}}{I-P_{\mathrm{j}}}
$$

where $W$ is the chip rate, $P_{\mathrm{j}}$ is the received signal power from user, $v_{\mathrm{j}}$ is the activity factor of user, $R_{\mathrm{j}}$ is the bit rate of user, and $I$ is the total received interference in the base station. Solving for $P_{\mathrm{j}}$ gives:

$$
P_{\mathrm{j}}=\frac{1}{1+\frac{W}{\left(E_{\mathrm{b}} / N_{\mathrm{o}}\right) \times R_{\mathrm{j}} \times v_{\mathrm{j}}}} \times I
$$


Since $P_{\mathrm{j}}=L_{\mathrm{j}} \times I$, the load factor of one connection can be obtained:

$$
L_{\mathrm{j}}=\frac{1}{1+\frac{W}{\left(E_{\mathrm{b}} / N_{\mathrm{o}}\right) \times R_{\mathrm{j}} \times v_{\mathrm{j}}}} .
$$

Additionally, the interference $i$, from the other cells must be taken into account:

$$
i=\frac{I_{\text {other cell }}}{I_{\text {own cell }}}
$$

where $I_{\text {other cell }}$ is other cells interference and $I_{\text {own cell }}$ is own cell interference. The uplink load factor can now be written as:

$$
\begin{aligned}
& \eta_{\mathrm{uL}}=(1+i) \times \sum_{j=1}^{M} L_{\mathrm{j}}= \\
& =(1+i) \times \sum_{j=1}^{M} \frac{1}{1+\frac{W}{\left(E_{\mathrm{b}} / N_{\mathrm{o}}\right) \times R_{\mathrm{j}} \times v_{\mathrm{j}}}}
\end{aligned}
$$

Statistical analysis from live network gives result that satisfying call setup success rate (CSSR) can be achieved with $\eta_{\mathrm{UL}}=0.98$ due to admission and congestion procedures specified in [17]. From (4), calculated maximum noise rise $\left(N R_{\max }\right)$ is then $16.99 \mathrm{~dB}$. From (1), UL RSSI $\max$ value is then $-89.91 \mathrm{dBm}$.

On the other hand, theoretical maximum uplink capacity, in term of bit rate, over bandwidth of $5 \mathrm{MHz}$ in WCDMA system is $5.76 \mathrm{Mbit} / \mathrm{s}$. This can be achieved with enhanced uplink (EUL) data transmission with time to transmit interval (TTI) of $2 \mathrm{~ms}$, specified in [18].

Investigation in lab environment has been performed by changing sirMax and TTE settings on RNC, which are more in detail explained at Tab. 1. EUL 2 ms capable device has been used in lab experiment, thus sirMax setting has been changed by sirMaxTti2 parameter, while transmissionTargetErrorTti2 parameter has been used to change TTE setting. From Fig. 1, if TTE is not OK, uplink outer loop power control will increase SIR target. TTE evaluation result is not OK if amount of transmitted data from mobile station is larger than amount of received data on RBS receiver. In lab experiment that condition has been executed by creating uplink load larger than theoretical maximum uplink capacity of $5.76 \mathrm{Mbit} / \mathrm{s}$. Thus uplink outer loop power control requested repeatedly increase of SIR target. However, increase of uplink radiated power is not allowed if calculated SIR target is larger than sirMaxTti2 value.

Average uplink (UL) throughput and UL RSSI, during constant uplink load, have been recorded. Uplink load, generated by uplink data transmission, has been same for every test case. Uplink data transmission has been performed by user datagram protocol (UDP), described in [19]. During period of $60 \mathrm{~s}$, data has been transmitted from

\begin{tabular}{|c|c|c|}
\hline Parameter name & Parameter description & Parameter value \\
\hline sirMaxTti2 & $\begin{array}{c}\text { Maximum allowed SIR } \\
\text { target for EUL 2ms TTI } \\
\text { users }\end{array}$ & $\begin{array}{c}\text { Range: }-82 \text { to } 173 \\
\text { Resolution: } 1 \\
\text { Unit: } 0.1 \mathrm{~dB}\end{array}$ \\
\hline $\begin{array}{c}\text { Wanted percentage of } \\
\text { transmissionTarget- } \\
\text { ErrorTti2 }\end{array}$ & $\begin{array}{c}\text { wrongly transmitted (not } \\
\text { acknowledged) data } \\
\text { frames, for EUL } 2 \mathrm{~ms} \\
\text { TTI users. }\end{array}$ & $\begin{array}{c}\text { Range: } 1 \text { to } 500 \\
\text { Resolution: } 1 \\
\text { Unit: } 0.1 \%\end{array}$ \\
\hline
\end{tabular}

Tab. 1. Main system parameters used in lab investigation.

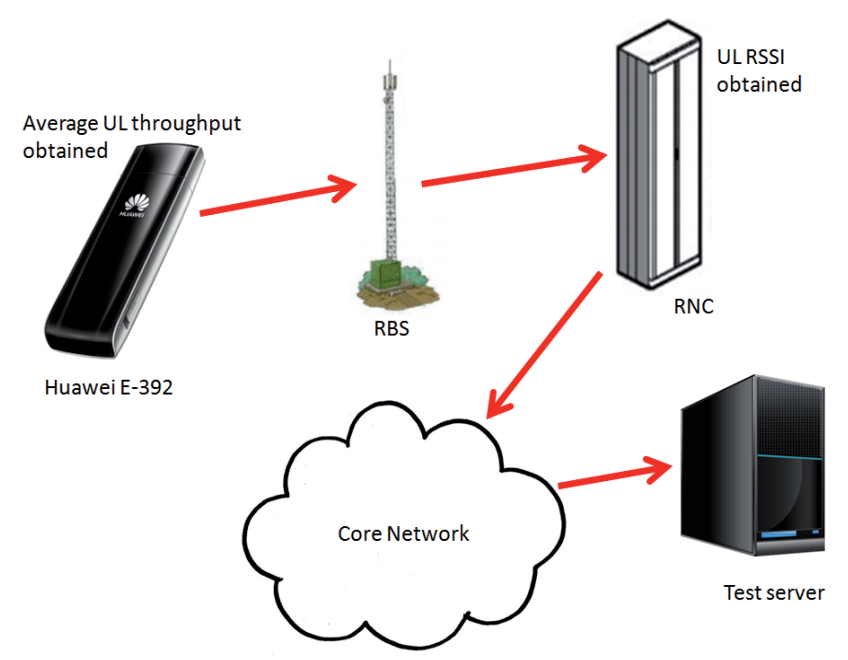

Fig. 2. Test case created and executed in lab environment.

the mobile station (USB stick Huawei E-392) to the test server. Average UL throughput over monitored $60 \mathrm{~s}$ has been obtained by DU-meter program, while average UL RSSI value has been obtained from RNC for monitored cell. Lab test case setup is presented at Fig. 2.

Two test cases have been executed:

- Changing sirMax value with constant TTE value of $2 \%$, which is default value recommended by vendor of mobile equipment.

- Changing TTE value with constant sirMax value of $13 \mathrm{~dB}$, which is default value recommended by vendor of mobile equipment.

\subsection{Changing sirMax Value with Constant TTE Value of $2 \%$} Fig. 4.

Results of this test case are presented at Fig. 3 and

From the obtained results it can be concluded that changing of sirMax value with constant TTE value of $2 \%$ has significant impact on UL RSSI and uplink throughput. The main reason is that changing of sirMax value directly influences maximum allowed radiated power for UE as described in Sec. 1. The more maximum radiated power has been limited by sirMax value, maximum achievable throughput and measured UL RSSI in the cell have been decreased. 


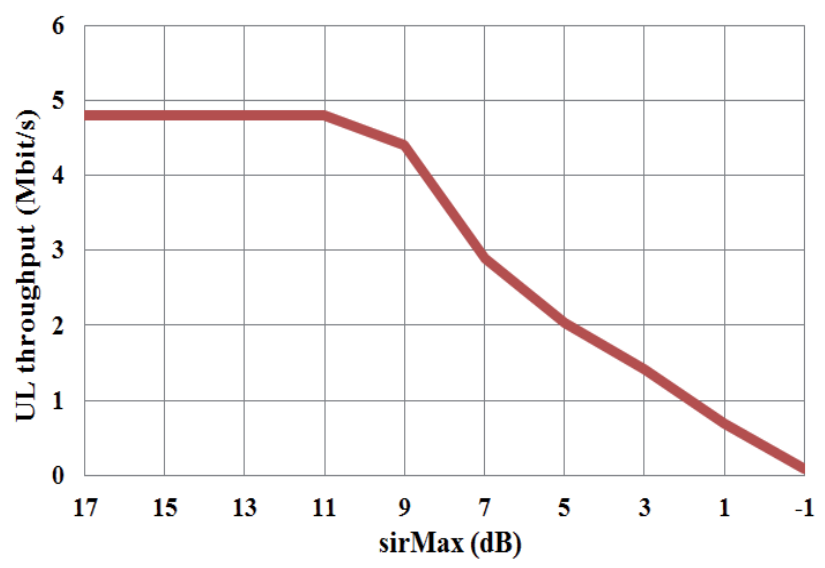

Fig. 3. Impact on uplink throughput by constant TTE value and variable sirMax value.

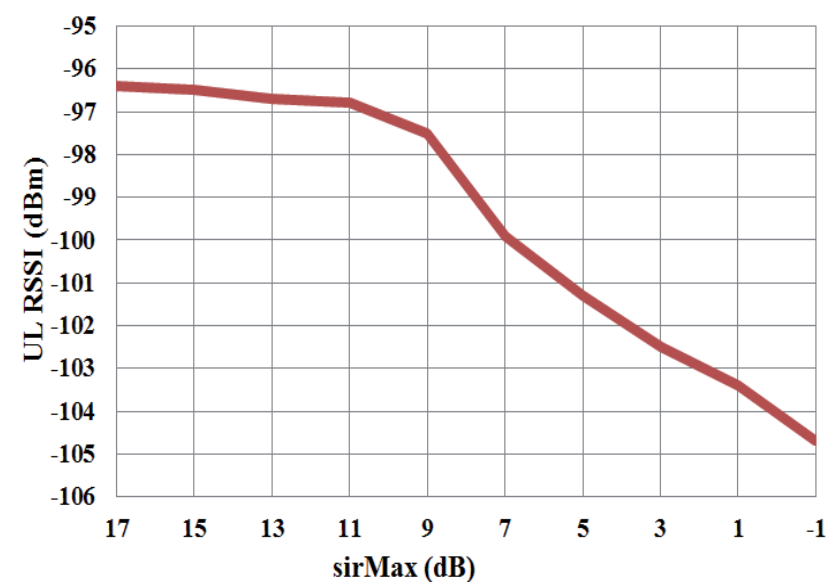

Fig. 4. Impact on UL RSSI by constant TTE value and variable sirMax value.

\subsection{Changing TTE Value with Constant sirMax Value of $13 \mathrm{~dB}$}

Results of this test case are presented at Fig. 5 and Fig. 6.

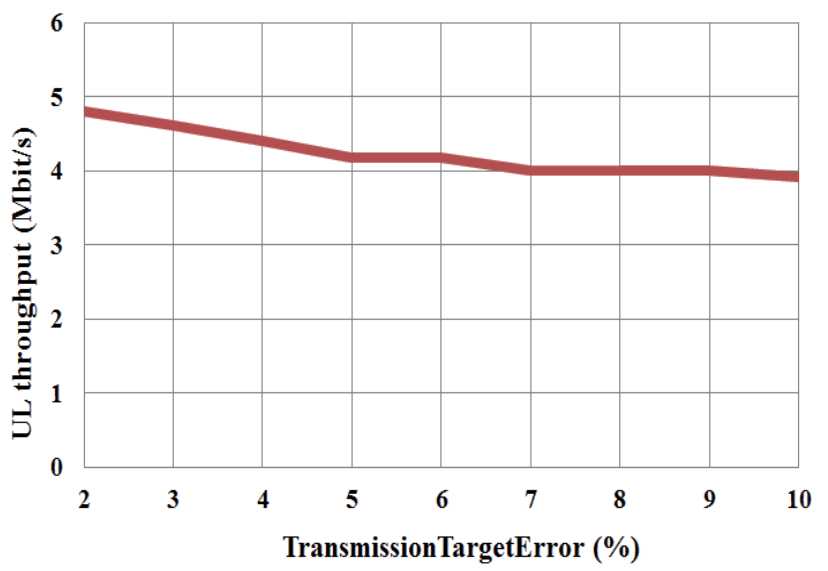

Fig. 5. Impact on uplink throughput by constant sirMax value and variable TTE value.

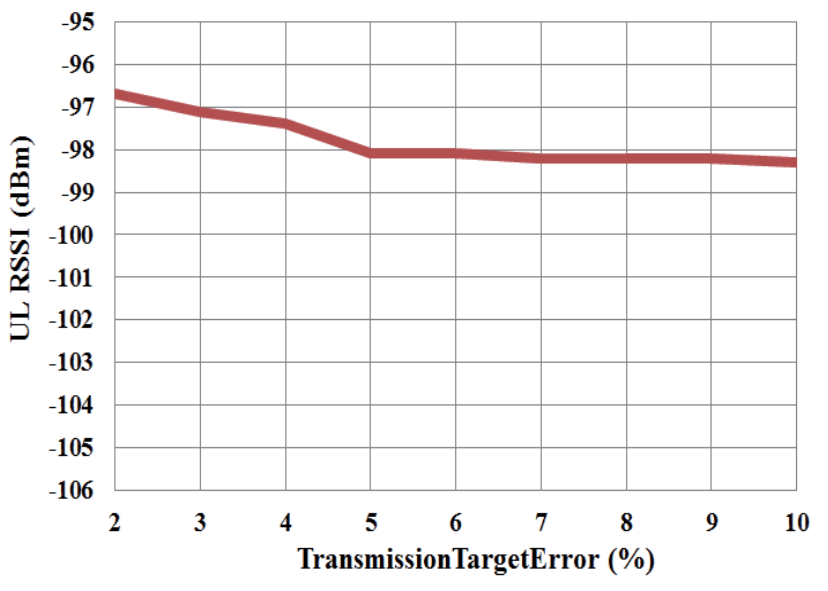

Fig. 6. Impact on UL RSSI by constant sirMax value and variable TTE value.

From the obtained results it can be concluded that changing of TTE value with constant sirMax value of $13 \mathrm{~dB}$ has almost no impact on UL RSSI and uplink throughput. The main reason is that sirMax value has been unchanged, thus maximum allowed radiated power has been same for all test cases, which was needed to upload data to test server with maximum throughput.

\subsection{Analysis of Recorded Results}

From the obtained results it can be concluded that out of two parameters used by uplink outer loop power control, sirMax has dominant impact on UL RSSI and uplink throughput. Depending on the uplink load of individual cells on the RBS there are two possible scenarios. First scenario is to set high value for sirMax. Benefit from this setting is that maximum uplink throughput for a single user in the cell would be provided. On the other hand, drawback of this setting is that in a multi user environment problem with uplink interference can occur that could lead to problems with CSSR. Second scenario is to set low value for sirMax. Benefit from this setting is that in a multi user environment there would be no problem with uplink interference and CSSR. On the other hand, in a low loaded cell, users would not be able to exploit entire cell capacity, thus they would not be able to achieve maximum uplink throughputs.

UL load on the cell differs not only from different cell to different cell, but also within the cell during the day, depending of number of users and their activity. Thus conclusion can be derived that it would be optimal if uplink outer loop power control would use sirMax that correspond to conditions on the uplink air interface at each cell, instead of using a fixed and same value for every cell whole time. Proposed idea how to achieve optimum uplink outer loop power control algorithm is dynamic changes of sirMax value based on uplink load on the cell. But prior to solution proposal, some real problem examples from live network has been presented in the next section. 


\section{Air Interface Uplink Capacity Overload Examples}

Problems with uplink air interface capacity, from live network, can be divided in 3 groups:

- Uplink air interface overload due to large number of simultaneous users, like concerts, sport events, traffic jams on highway etc.

- Uplink air interface overload caused from outer source of interference presented at [20]. Sources of outer interference could be malfunctioning radio devices (TV antenna amplifier, DECT systems, FAX devices etc.), mobile operators from neighboring countries that use same frequency spectrum, wrongly installed components (power tappers and splitters) at indoor distributed antenna systems.

- Uplink air interface overload caused by passive inter modulation (PIM) products created by own system. PIM has become a significant factor in the recent times when the amount of frequency spectrum used by each operator has increased significantly by introducing an increasing number of $5 \mathrm{MHz}$ band-carriers (WCDMA), as well as new broadband technologies like long term evolution (LTE). Passive inter-modulation occurs when two or more signals are present in passive device (cable, connector, isolator, switch, splitter, tapper or antenna) and this device exhibits a non-linear response [21].

At Fig. 7 an example of uplink air interface overload during football match has been presented. Presented results at Fig. 7 indicate that impact on uplink capacity is greatest just before match and during half time. Overload during half time has been so high that almost every service establishment attempt was unsuccessful.

UL RSSI increase at Fig. 7 has been caused by large number of simultaneous users that were trying to access cell at the same time. Simultaneous radiated power in uplink direction from large number of users cause severe

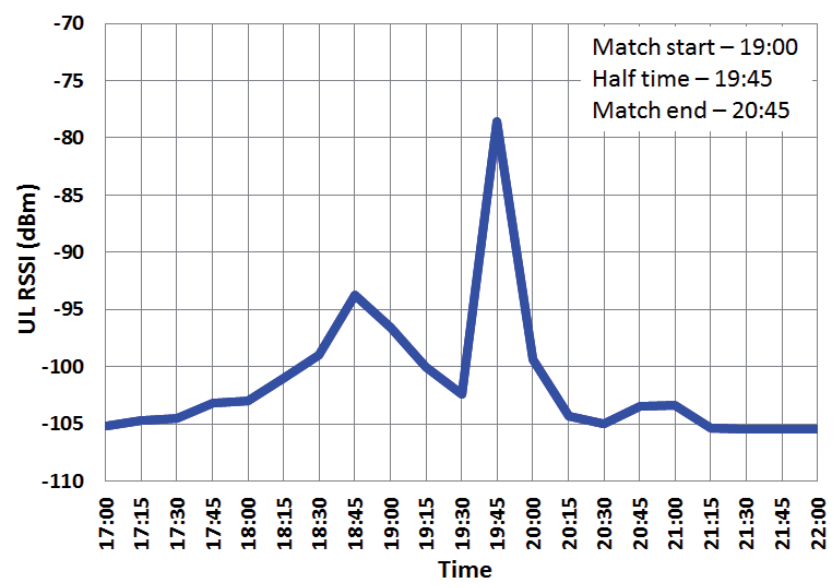

Fig. 7. Impact on UL RSSI at one WCDMA cell during football match. interference [16]. Thus number of wrongly transmitted blocks increases dramatically and uplink outer loop power control increases SIR target to satisfy TTE value set on RNC. By doing that all users in the cell are instructed to increase radiated power, thus increasing UL RSSI.

UL RSSI value at Fig. 7 has been obtained by statistical observability through RNC performance management counters pmSumUlRssi and pmSamplesUlRssi that have been more in detail explained in Sec. 5.

At presented example uplink performance has been strongly degraded, up to the point that every service establishment attempt was unsuccessful. Main reason for that is not only in problem causes, but in fact that uplink outer loop power control could not adapt to new situation. Instead to ask for higher SIR target due to unsatisfied TTE condition it should limit radiated power by limiting sirMax value according to condition on uplink air interface. With proposed unique sirMax value per cell, balanced situation could be achieved. Maximum user's performance would be limited, but also maximum noise rise caused by single user would be limited, according to given uplink load. To verify proposed idea, simulation has been created and executed that is presented in next section.

\section{Simulation of Proposed Change in sirMax Implementation}

Simulation has been created and executed in commercial radio planning tool, that use Monte Carlo algorithm, which simulates the way a network allocates resource units to users accessing different services [22]. It is possible to import real network traffic to radio planning tool, obtained by performance management counters on RNC. Worst case scenario has been created that maximum uplink load during day for each cell has been imported to radio planning tool.

This is for macro sites very rare case that all cells have maximum load at exactly same point in time. It actually corresponds to overload scenario due to large number of simultaneous users, where all cells do have maximum load at exactly same time. Thus created simulation corresponds to some real case overload scenario during concert or sport event. Common sirMax for all cells used in simulation was $11 \mathrm{~dB}$, which is optimized value by network operator for normal cell load. Two test simulations have been executed on cluster of 9 RBS with 27 cells.

- Test case 1 - sirMax value is identical $(11 \mathrm{~dB})$ for all cells in the cluster.

- Test case 2 - sirMax value is unique per cell depending of uplink load. Unique value of sirMax per cell has been obtained from (12).

In both test cases used TTE value was common for all cells and equal to $3.5 \%$, which is optimized value by network operator for normal cell load. Results of simulations are presented at Tab. 2 . 


\begin{tabular}{|c|c|c|c|}
\hline & $\begin{array}{c}\text { Number of } \\
\text { users }\end{array}$ & Blocked users & CSSR (\%) \\
\hline Test case 1 & 864 & 42 & 95.14 \\
\hline Test case 2 & 864 & 8 & 99.07 \\
\hline
\end{tabular}

Tab. 2. Simulation results for common sirMax implementation for all cells against unique sirMax implementation per cell respectively to uplink load.

As it can be concluded from results of simulations, significant improvement has been recorded with unique sirMax implementation per cell. As it was explained in Sec. 2, uplink load on the cell differs not only from different cell to different cell but also within the cell during the day. Therefore, it is also recommended that sirMax is not only unique per cell, but that is unique within cell during different period of time. Proposal how to achieve it has been presented in the next section.

\section{Design of Self-optimizing Uplink Outer Loop Power Control}

Design of self-optimizing uplink outer loop power control is based on fact that RNC is informed by each cell of cell's current UL RSSI. How often RNC is informed about UL RSSI, can be presented through RNC performance management counters that exist for every cell controlled by RNC:

- pmSumUlRssi is the counter that records the value of received total wideband power (RTWP) measured on the cell and sent to the RNC via nodeB application part (NBAP) common measurement reporting [23], over Iub interface which is specified in [24], where RTWP refers to UL RSSI measurement.

- pmSamplesU1Rssi is a counter that records how often sample has been recorded and it is actually captured every $10 \mathrm{~s}$. That means that RNC is notified every $10 \mathrm{~s}$ of UL RSSI measured at each cell on RNC.

Design of self-optimizing uplink outer loop power control consists of introduction of new parameter on RNC, which would be used to derive sirMax value. By doing so sirMax would not be any more fixed value, but it would be derived from combination of new parameter and current UL RSSI on the cell. New proposed parameter would define maximum allowed UL RSSI on the cell, thus parameter has been called MaxULRSSI. Then sirMax value would be calculated as:

$\operatorname{sirMax}[\mathrm{dB}]=\operatorname{Max} U L R S S I[\mathrm{dBm}]-U L \operatorname{RSSI}[\mathrm{dBm}]+C[\mathrm{~dB}]$

where sirMax is the value used by uplink outer loop power control, MaxULRSSI is maximum allowed UL RSSI on the cell, UL RSSI is measured UL RSSI on the cell and $C$ is constant that would be used by operator to modify dependency of sirMax to measured UL RSSI on the cell. Proposed changes in current uplink outer loop power control to achieve self-optimizing uplink outer loop power control are presented in Fig. 8:

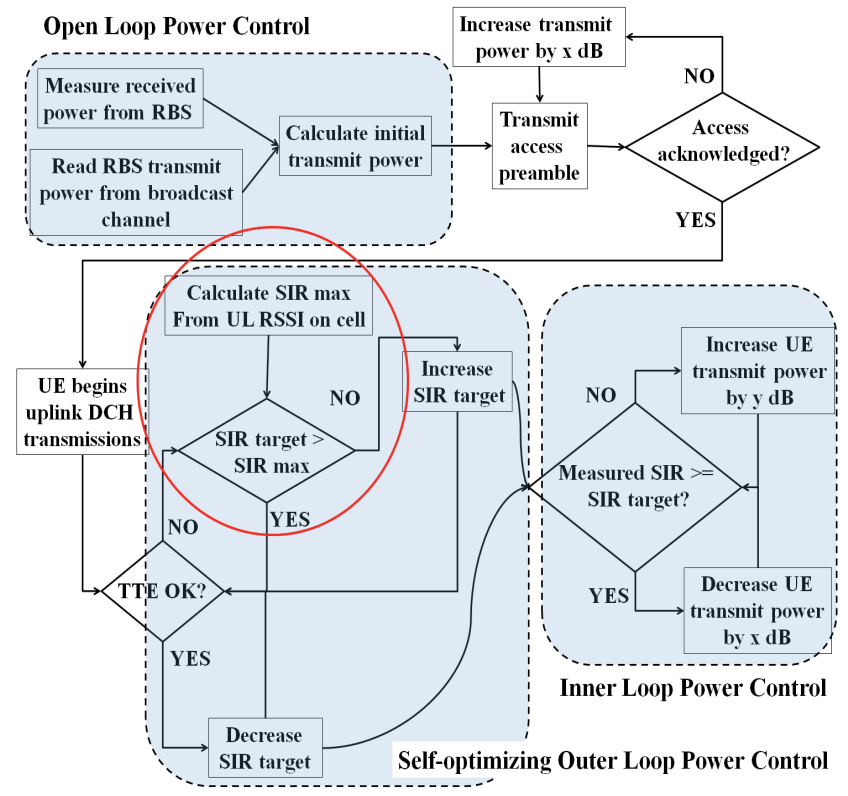

Fig. 8. Cooperation between three types of uplink power control with proposed changes for outer loop power control.

Proposal for MaxULRSSI is to be fixed value and that it is equal $-90 \mathrm{dBm}$, which correspond to $\eta_{\mathrm{UL}}=0.98 \mathrm{de}-$ scribed in Sec. 2. If sirMax would be derived by (12), it is obvious that sirMax then would directly depend on measured UL RSSI value. In that case uplink outer loop power control would self-optimize used sirMax value based on current uplink load on the cell. Example of calculated sirMax value by self-optimizing uplink outer loop power control with MaxULRSSI $=-90 \mathrm{dBm}$ and $C=0 \mathrm{~dB}$ is given in Tab. 3. To compare sirMax used by self-optimizing uplink outer loop power control to sirMax used by current uplink outer loop power control, simulation has been executed for one real macro cell from the previous section, which UL RSSI value during day is presented in Fig. 9.

\begin{tabular}{|c|c|}
\hline Measured UL RSSI (dBm) & sirMax (dB) \\
\hline-107 & 17 \\
\hline-106 & 16 \\
\hline-105 & 15 \\
\hline-104 & 14 \\
\hline-103 & 13 \\
\hline-102 & 12 \\
\hline-101 & 11 \\
\hline-100 & 10 \\
\hline-99 & 9 \\
\hline-98 & 8 \\
\hline-97 & 7 \\
\hline-96 & 6 \\
\hline-95 & 5 \\
\hline-94 & 4 \\
\hline-93 & 3 \\
\hline-92 & 2 \\
\hline-91 & 1 \\
\hline-90 & 0 \\
\hline-89 & -1 \\
\hline-88 & -2 \\
\hline-87 & -3 \\
\hline & \\
\hline
\end{tabular}

Tab. 3. Calculated sirMax by self-optimizing uplink outer loop power control according to measured UL RSSI. 


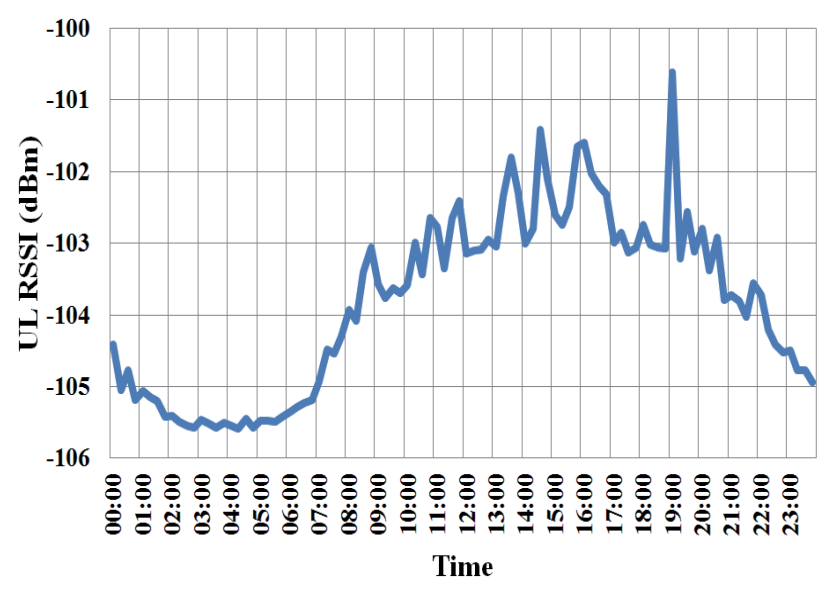

Fig. 9. UL RSSI distribution during day for one WCDMA cell.

From the results of simulation, which are presented at Fig. 10, it can be concluded that self-optimizing uplink outer loop power control has adapted to the uplink load on the cell. Other important result that can be concluded is that new design would not only limit interference during high load by decreasing sirMax value, but would actually allow higher radiated power from mobile stations during low load on the cell. This would be done by increasing sirMax value, thus allowing higher uplink throughputs. On the other hand, current uplink outer loop power control, regardless of uplink load, is using same sirMax value throughout whole time.

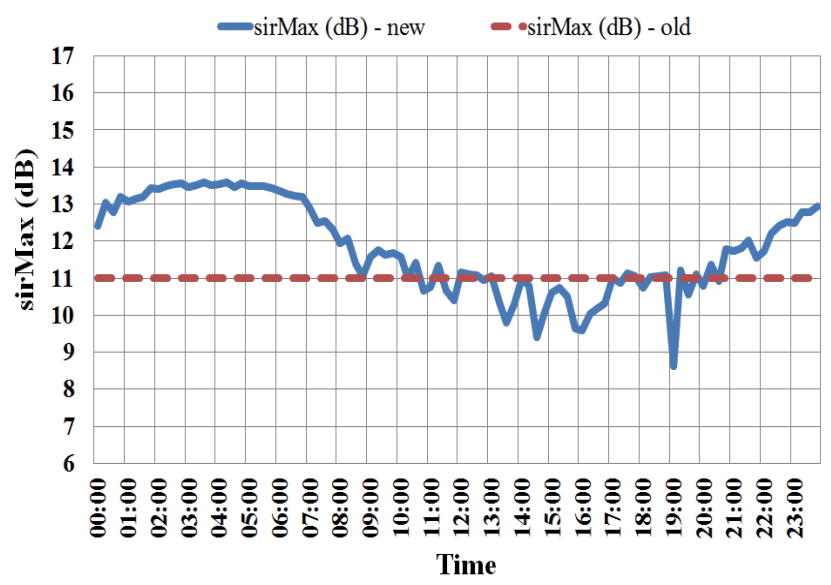

Fig. 10. SirMax used by uplink outer loop power control: comparison between current implementation and new design

The most important part regarding critical scenarios described at Sec. 3 is in fact that self-optimizing uplink outer loop power control would use extremely low values of sirMax for cells with high UL RSSI, thus interference would be limited. Still connections would be possible with low uplink throughput (from Fig. 3, for sirMax $=-1 \mathrm{~dB}$, uplink throughput per user is up to $90 \mathrm{kbit} / \mathrm{s}$ ). That would be significant improvement compared to presented scenarios from Sec. 3.

To verify possibility of implementation of new approach, program has been created and executed, that was reading performance management statistics (aggregated UL RSSI value over $15 \mathrm{~min}$ ) for one cell in lab environment. Based on obtained result from statistic, program is running shell scripts on Operation Support System (OSS), which via Advanced Managed Object Scripting (AMOS) are changing sirMax value on RNC. Results presented at Tab. 3 were successfully obtained for given test cell.

\section{Conclusion}

In this paper proposal for completely new approach in resolving problem with WCDMA uplink capacity on air interface has been presented. Proposed solution is to turn the problem solving on RNC level and to improve uplink outer loop power control. Presented simulation results in Sec. 4 and proposal for new design of uplink outer loop power control, that is dependent of uplink load, lead to conclusion that uplink interference could be contained to some sustainable level. A novel self-optimizing uplink outer loop power control mechanism for WCDMA network, presented in Sec. 5, could bring final solution for overload scenarios described in Sec. 3. Additionally, proposed solution could bring better QoS during normal uplink load on the cell.

\section{References}

[1] SONG, L., MANDAYAN, N. B., GAJIC, Z. Analysis of an up/down power control algorithm for the CDMA reverse link under fading. IEEE Journal on Selected Areas in Communications, 2001, vol. 19 , no. 2 , p. 277-286. DOI: 10.1109/49.914505

[2] KAUR KHANGURA, S., KAUR, K., UPPAL, R. S. Power control algorithms in wireless communication. International Journal of Computer Applications, 2010, vol. 1, no. 12, p. 82-87. DOI: 10.5120/253-410

[3] GOLDSMITH, A. Wireless Communication. Cambridge, 2005. ISBN: 0521837162, 9780521837163

[4] 3rd Generation Partnership Project (3GPP), Technical Specification (TS) 25.401 V12.0.0 (2013-12). UTRAN Overall Description

[5] 3rd Generation Partnership Project (3GPP), Technical Specification (TS) 25.427 V12.1.0 (2014-09). UTRAN Iub/Iur Interface User Plane Protocol for DCH Data Streams

[6] KAARANEN, H. UMTS Networks: Architecture, Mobility and Services. Wiley, 2005. ISBN: 0470011033, 9780470011034

[7] 3rd Generation Partnership Project (3GPP), Technical Specification (TS) 25.214 V12.0.0 (2014-09). Physical Layer Procedures (FDD)

[8] CHEVALliER, C., BRUNNER, C., GARAVAGLIA, A. MURRAY, K. P., BAKER, K. R. WCDMA (UMTS) Deployment Handbook: Planning and Optimization Aspects. Wiley, 2006. ISBN: 0470035730, 9780470035733

[9] KEJIK, P., HANUS, S. Enhanced receivers for interference cancellation in $3 \mathrm{G}$ systems. Radioengineering, 2009, vol. 18, no. 4, p. 477-484 DOI: $10.13164 / \mathrm{re}$

[10] TOLLI, A., HOLMA, H. Simulated and measured performance of 4-branch uplink reception in WCDMA. In Proceedings of the $53^{\text {rd }}$ 
Vehicular Technology Conference (VTC). Greece, 2001, p. 2640 to 2644. DOI: 10.1109/VETECS.2001.944079

[11] PUSNIK, T., SKOK, G., IVANDIC, I. High capacity event network planning - Poljud. In Proceedings of the $56^{\text {th }}$ International Symposium ELMAR-2014. Zadar (Croatia), 2014, p. 63-67. DOI: 10.1109/ELMAR.2014.6923316

[12] HONG-NING, D., KAM-WING, N., MINGLU, L., MIN-YOU, W. An overview of using directional antennas in wireless networks. International Journal of Communication Systems, 2011, vol. 26, no. 4, p. 413-448. DOI: 10.1002/dac.1348

[13] LANER, M., SVOBODA, P., RUPP, M. Outer-loop power control in a live UMTS network: measurement, analysis and improvement In Proceedings of the $4^{\text {th }}$ International Symposium on Communications, Control and Signal Processing (ISCCSP-2010). Limassol (Cyprus), 2010, p. 1-5. DOI: 10.1109/ISCCSP.2010.5463465

[14] RANHEMA, M. UMTS Network Planning, Optimization, and Inter-Operation with GSM. Wiley, 2008. ISBN: 047082302X, 9780470823026

[15] ALAMI, F. T., AKNIN, N., EL MOUSSAOUI, A. Capacity estimation of multi-service cellular network. International Journal of Computer Science and Engineering (IJCSE), 2011, vol. 3, no. 3, p. 1363-1368. ISSN: 0975-3397.

[16] HOLMA, H., TOSKALA, A. WCDMA for UMTS: HSPA Evolution and LTE. Wiley, 2010. ISBN: 1119991900, 9781119991908

[17] 3rd Generation Partnership Project (3GPP), Technical Specification (TS) 25.922 V7.1.0 (2007-03). Radio Resource Management Strategies

[18] 3rd Generation Partnership Project (3GPP), Technical Specification (TS) 25.922 V12.1.0 (2014-09). Enhanced Uplink; Overall Description; Stage 2

[19] SHANMUGAM, R., PADMINI, R., NIVEDITA, S. Special Edition Using TCP/IP. Que Publishing, 2002. ISBN: 0789727099, 9780789727091

[20] KLOZAR, L., POLAK, L., KALLER, O., PROKOPEC, J. Effect of co-existence interferences on QoS of HSPA/WCDMA mobile networks. In $23^{\text {rd }}$ International Conference on Radioelektronika 2013. Pardubice (Czech Republic), 2013, p. 312-315. DOI: 10.1109/RadioElek.2013.6530937
[21] TOLSTRUP, M. Indoor Radio Planning: A Practical Guide for GSM, DCS, UMTS, HSPA and LTE. Wiley, 2011. ISBN: 1119973686, 9781119973683

[22] Atoll RF planning and optimization software. Atoll 2.8 .1 Measurements and Model Calibration Guide. Forsk, 2009

[23] 3rd Generation Partnership Project (3GPP), Technical Specification (TS) 25.433 V12.2.0 (2014-09). UTRAN Iub Interface Node B Application Part (NBAP) Signaling

[24] 3rd Generation Partnership Project (3GPP), Technical

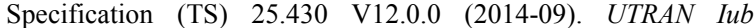
Interface General Aspects and Principles

\section{About the Authors ...}

Angelo Gary MARKOČ was born in Euclid, USA in 1982. He received his B.Sc. degree in Electrical Engineering from the Faculty of Electrical Engineering and Computing, University of Zagreb, Croatia in 2005. He is a frequency and parameterization planning expert in Radio Access Network Development Department at the Vipnet d.o.o. in Zagreb. His main research interests are in testing and implementation of new WCDMA and LTE functionalities with special focus in creating and designing algorithms that automatically optimize network performances based on inputs collected from live network. He published several papers in national and international conferences.

Gordan ŠIŠUL received B.Sc., M. Sc. and Ph.D. in Electrical Engineering from the Faculty of Electrical Engineering, University of Zagreb, Croatia in 1996, 2000 and 2004 respectively. He is currently employed as an Associate Professor at the same Faculty. His academic interests include wireless communications, signal processing applications in communications, modulation techniques and radio propagation. 